\title{
Malignant Hypertension Associated with Obstructive Hydrocephalus
}

\author{
-Case Report- \\ Hideki NAKANO, Yoshiyuki TOMITA*, Kuniaki BANDOH, and Makoto MIYAOKA \\ Department of Neurosurgery, Fujisawa City Hospital, Fujisawa, Kanagawa; \\ ${ }^{*}$ Department of Neurosurgery, Juntendo University Hospital, Tokyo
}

\begin{abstract}
A 36-year-old male presented with headache, vomiting, and gait disturbance. Examination found marked anemia, renal failure, markedly choked disks, and hypertensive encephalopathy. Magnetic resonance imaging demonstrated diffuse swelling of the brainstem and cerebellum, and obstructive hydrocephalus. Treatment with steroid, glycerol, and antihypertensive drugs resulted in a slow decrease in the brain swelling and cerebral edema. However, hydrocephalus and intracranial hypertension persisted, requiring a shunt operation. Hypertensive encephalopathy is usually improved by the treatment of hypertension, but shunt operation may be required to treat exacerbated intracranial pressure associated with obstructive hydrocephalus.
\end{abstract}

Key words: malignant hypertension, hypertensive encephalopathy, hydrocephalus, renal failure

\section{Introduction}

Malignant hypertension is associated with hypertensive encephalopathy, retinal hemorrhage with papilledema, and renal failure, as well as severe hypertension. ${ }^{10)}$ Renal failure, cerebral hemorrhage, and congestive heart failure often prove fatal if the blood pressure is not controlled appropriately. ${ }^{10)}$ The main symptoms of hypertensive encephalopathy include headache, vomiting, visual disorders, and disturbance of consciousness, and are caused by vasospasm and edema of the cerebral tissue. Neuroimaging demonstrates severe brain swelling, narrowing of the cerebral sulci, and compression of the cerebral ventricles.

We describe a rare case of malignant hypertension associated with obstructive hydrocephalus due to brainstem swelling.

\section{Case Report}

A 36-year-old male visited our hospital on July 22 , 1994 complaining of headache and vomiting. He had received oral medication for schizophrenia di-

Received March 18, 1996; Accepted November 15, 1996 agnosed 5 years previously, but had stopped taking the medication after 3 years. He had no history of hypertension, diabetes mellitus, or renal disease.

Physical examination on admission found he was thin, with blood pressure of $230 / 100 \mathrm{mmHg}$, pulse rate of $104 / \mathrm{min}$, and body temperature of $36.2^{\circ} \mathrm{C}$. No chest or abdominal abnormalities were detected, and no edema was seen in any extremity. His consciousness was slightly disturbed, and he was drowsy. When he was awake, contact was poor, and he tended to be abulic and autistic. Bilateral visual acuity was markedly reduced, and severe papilledema and bleeding were present (Fig. 1). Muscle strength was not reduced, and the deep tendon reflexes in the extremities were normal. Trunkal ataxia was present, and he could not walk unassisted.

Hematological examination on admission revealed red blood cells of $204 \times 10^{4} / \mu$, hemoglobin $6.6 \mathrm{~g} / \mathrm{dl}$, hematocrit $18.2 \%$, white blood cells $4900 / \mu \mathrm{l}$ (84\% neutrophils, $12 \%$ lymphocytes, $3 \%$ monocytes), and platelet count $3.8 \times 10^{4} / \mu \mathrm{l}$, indicating marked anemia. Blood chemistry examination found total protein $6.7 \mathrm{~g} / \mathrm{dl}$, blood urea nitrogen $149 \mathrm{mg} / \mathrm{dl}$, creatinine $17.0 \mathrm{mg} / \mathrm{dl}$, Na $137 \mathrm{mEq} / \mathrm{l}, \mathrm{K} 3.4 \mathrm{mEq} / \mathrm{l}, \mathrm{Cl}$ $98 \mathrm{mEq} / \mathrm{l}$, total cholesterol $159 \mathrm{mg} / \mathrm{dl}$, blood sugar $112 \mathrm{mg} / \mathrm{dl}$, glutamic oxaloacetic transaminase $21 \mathrm{IU} /$ 


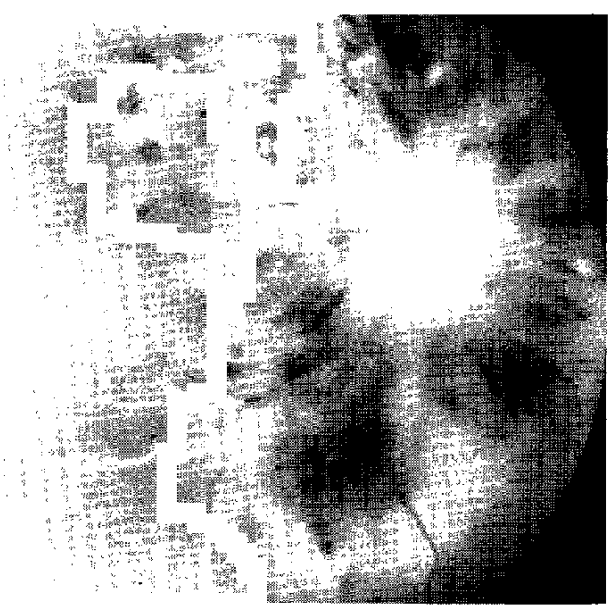

Fig. 1 Optic funduscopy image showing marked choked disk and retinal hemorrhage.
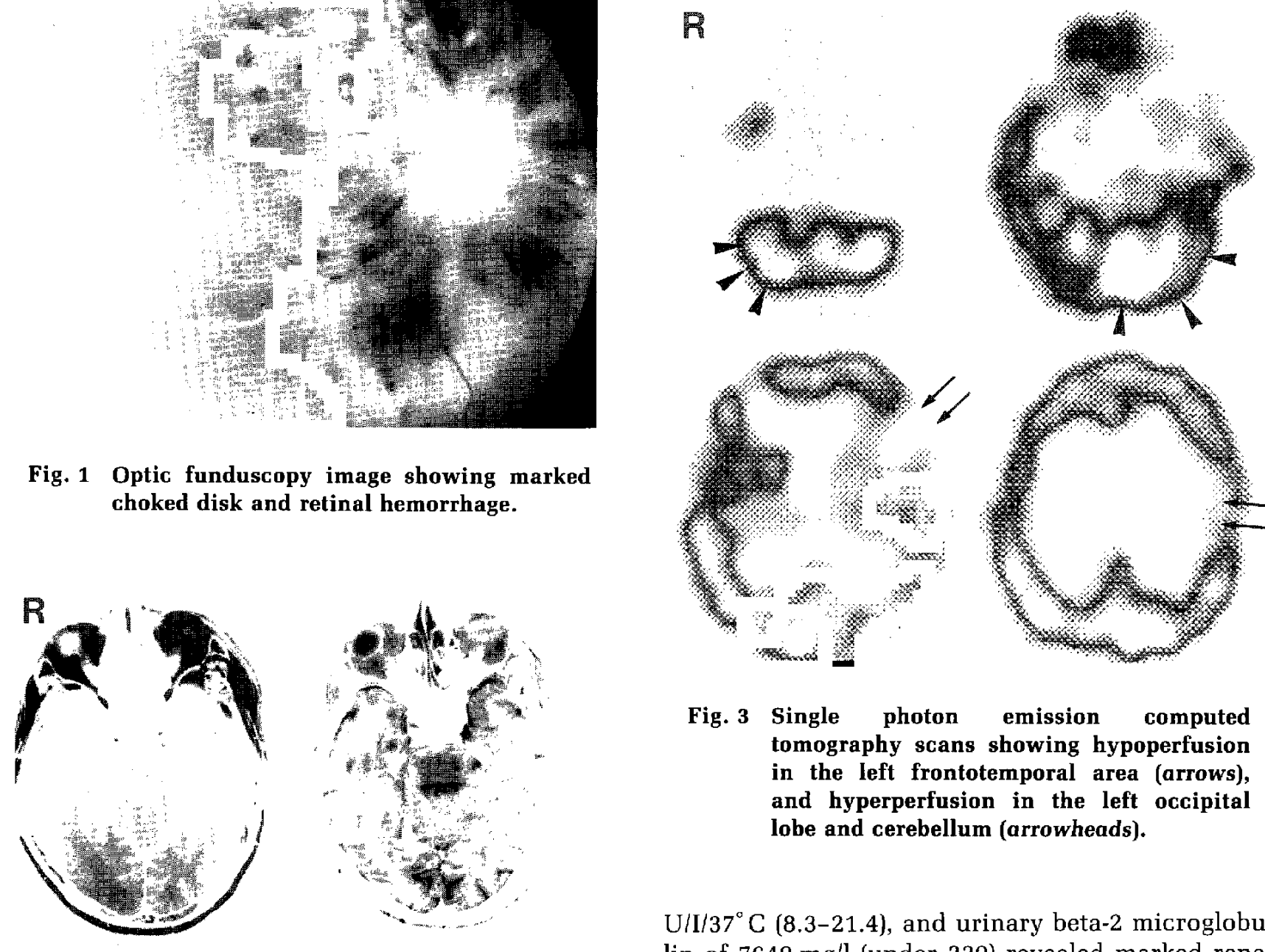

Fig. 3 Single photon emission computed tomography scans showing hypoperfusion in the left frontotemporal area (arrows), and hyperperfusion in the left occipital lobe and cerebellum (arrowheads).

$\mathrm{U} / \mathrm{I} / 37^{\circ} \mathrm{C}(8.3-21.4)$, and urinary beta-2 microglobulin of $7640 \mathrm{mg} / \mathrm{l}$ (under 230) revealed marked renal dysfunction.
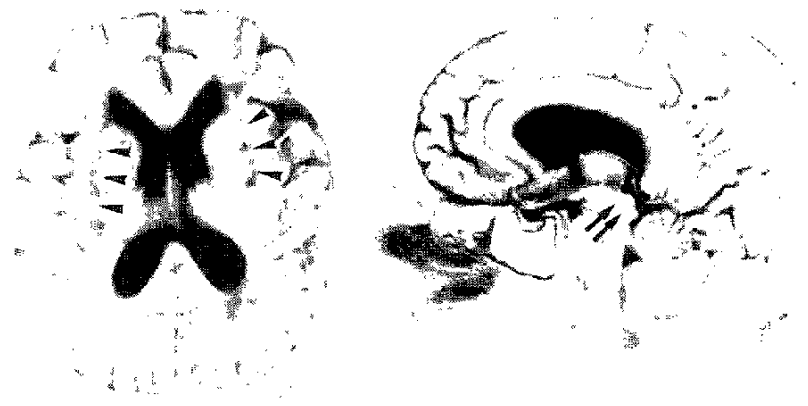

Fig. 2 Magnetic resonance images showing swelling in the cerebellum and brainstem. The aqueduct is compressed and barely visible (arrows). Enlarged lateral ventricles and multiple high intensity areas are noted on the $T_{2}$-weighted image (arrowheads).

l, glutamic pyruvic transaminase $10 \mathrm{IU} / \mathrm{l}$, and lactate dehydrogenase $1848 \mathrm{IU} / \mathrm{l}$. Renin activity of $19 \mathrm{ng} / \mathrm{ml} /$ $\mathrm{hr}$ (normal range 0.3-2.9), aldosterone of $640 \mathrm{pg} / \mathrm{ml}$ [29.9-159], angiotensin converting enzyme of 6.31
Chest radiography on July 22, 1994 revealed his cardiothoracic ratio had increased to $62 \%$. Computed tomography (CT) revealed marked swelling of the cerebellum and brainstem, appearing as a low density area in the brainstem, and acute hydrocephalus. $\mathrm{T}_{2}$-weighted magnetic resonance (MR) imaging indicated high intensity areas in the pons and midbrain tegmentum, and marked swelling was noted in the cerebellum and brainstem. The lateral ventricles were enlarged and multiple high intensity areas were seen in the cerebral white matter (Fig. 2).

Hemodialysis three times per week to treat renal failure was commenced on July 25, 1994, and antihypertensive agent (nicardipine) was administered. There was a gradual reduction in blood pressure. Single photon emission computed tomography (SPECT) with technetium-99m-hexamethyl propyleneamine oxime on July 27,1994 indicated increased perfusion in the posterior cranial fossa and occipital lobe, and a region of low perfusion extending from the left frontal lobe through the temporal lobe (Fig. 3). MR imaging on July 28, 1994 indicated 

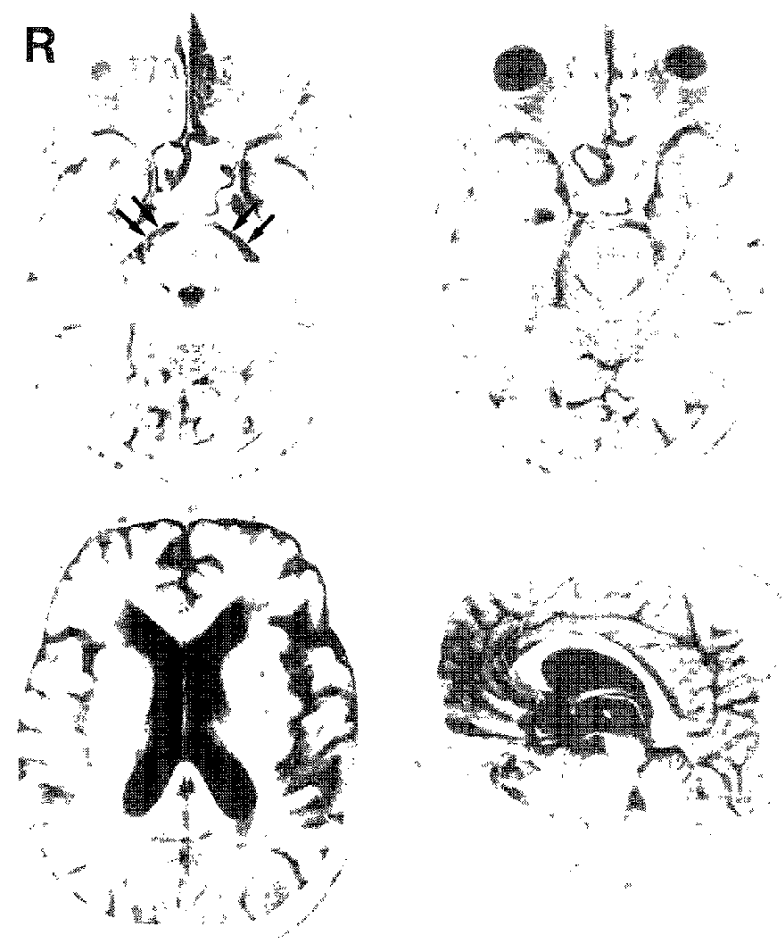

Fig. $4 \mathbf{T}_{2}$-weighted magnetic resonance images showing slight improvement in the swelling of the cerebellum and brainstem. The cerebellopontine and prepontine cisterns are visible (arrows), but ventricular dilation persists.

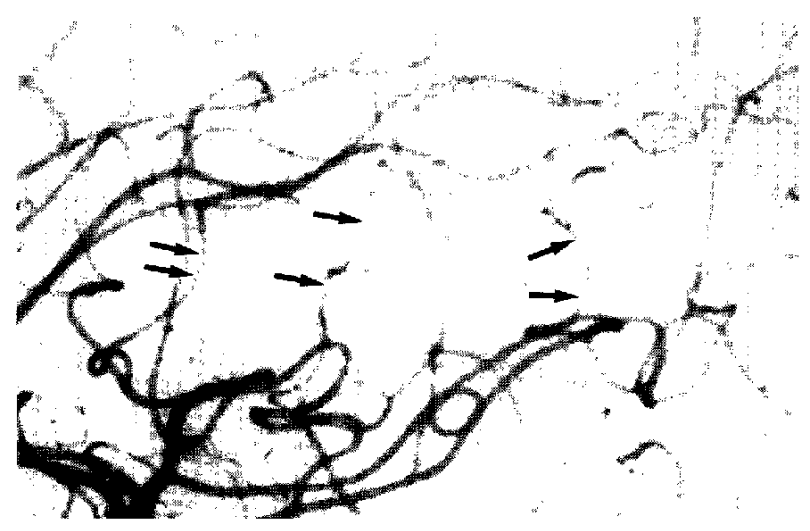

Fig. 5 Cerebral angiogram showing multiple segmental vasospasm [arrows] in the branches of the middle cerebral artery.

slight improvements in the swelling of the cerebellum and brainstem, and the basal cistern was delineated, but enlargement of the ventricular system persisted (Fig. 4). The patient was still drowsy,
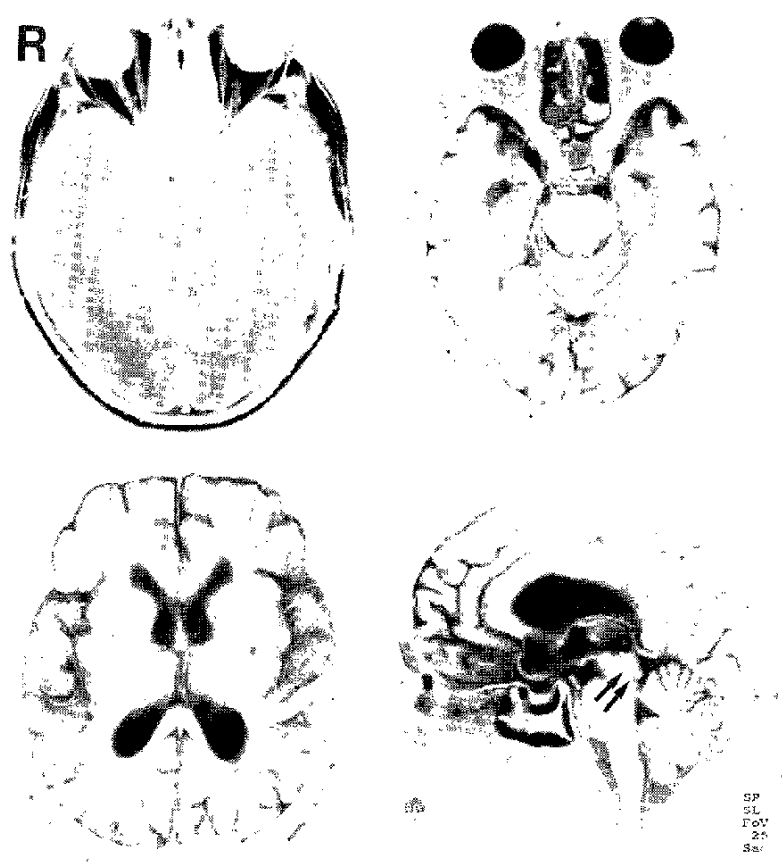

Fig. 6 Magnetic resonance images showing normal size and intensity of the cerebellum and brainstem. The aqueduct is normal (arrows) and hydrocephalus is improved.

and visual disturbance continued.

Lumbar puncture on July 29, 1994 found intracranial pressure (ICP) was over $300 \mathrm{mmH}_{2} \mathrm{O}$. Therefore, intracranial hypertension still remained, despite the slight decrease in the edema and swelling in the brainstem and cerebellum. Extraventricular drainage was performed on July 29, 1994, to reduce pressure but avoid a sudden reduction. Cerebral angiography (August 2, 1994) indicated no abnormal findings in the vertebrobasilar artery system, but multiple segmental vasospasms were observed in the anterior cerebral artery and the cortical branches of the middle cerebral artery (Fig. 5].

A shunt operation was performed on August 5, 1994. There was gradual improvement in consciousness disturbance and laboratory findings after the commencement of antihypertensive therapy and the shunt operation. On September 14, 1994, MR imaging revealed that the swelling and edema in the cerebellum and the brainstem, and hydrocephalus had disappeared (Fig. 6). His blood pressure became stabilized, headache and vomiting ceased, and visual acuity and walking were improved. He is now undergoing hemodialysis as an outpatient, and can lead a normal life independently. 


\section{Discussion}

The present patient suffered sudden onset of renal failure, hypertension, disturbance of consciousness, and retinal hemorrhage. Our diagnosis was malignant hypertension. Severe anemia, hypokalemia, and hyperaldosteronemia were all compatible with this diagnosis. Neurological symptoms of varying severity, such as disturbance of consciousness, convulsion, visual disturbance, headache, and vomiting can all result from hypertensive encephalopathy, but the causative mechanism is still not clear. Cerebral blood flow usually is kept constant within the range of autoregulation. Hypertensive encephalopathy is considered to occur when the systemic blood pressure rises above the autoregulatory range. ${ }^{\text {fi) }}$ This mechanism is supported by the observation of cerebral vasospasm ${ }^{7}$ and passive dilation in the arterioles. ${ }^{2)}$ Both prolonged severe ischemia due to vasospasm, and passive dilation due to malignant hypertension may result in leakage of protein and water from the blood vessels to the interstitial tissue. These phenomena are complementary, and appear simultaneously. CT, MR imaging, and SPECT findings in 14 patients with hypertensive encephlaopathy suggested that cerebral ischemia and excessive cerebral blood flow appear simultaneously in patients with hypertensive encephalopathy. ${ }^{51}$ In our patient, SPECT showed sites of both reduced and increased blood flow. Morphological changes in blood vessels due to spasms have also been found in animal experiments, ${ }^{4)}$ and cerebral angiography showed segmental stenosis and dilation in our patient.

Cerebral edema frequently occurs in supratentorial locations caused by hypertensive encephalopathy, but is also found in the region of the posterior circulation, especially in the posterior cranial fossa. ${ }^{3} \mathrm{MR}$ imaging is useful for the diagnosis and observation of the course of hypertensive encephalopathy because it tends to occur in the cerebellum and brainstem which are surrounded by bony structures, and edema of brain tissue can be sharply delineated. CT and MR imaging cannot differentiate localized or diffuse edema from tumors, cerebral infarction, or demyelinating disease. However, the findings of hypertensive encephalopathy are reversible and disappear when blood pressure is brought under control. ${ }^{3,5)}$ Malignant hypertension requires urgent treatment for hypertension and renal failure, but caution is required in treating hypertension because the lower limit of autoregulation may shift to a high value, and the presence of complications such as vasospasm, cerebral ischemia due to excessive falls in blood pressure, and cerebral infarction.
Previous patients with malignant hypertension have presented with high ICP, increases in brain weight due to cerebral edema, flattening of the sulci, and compression and shrinkage of the cerebral ventricles. ${ }^{1 j}$ However, a patient with malignant hypertension and hydrocephalus had expanded fourth and lateral ventricles thought to be caused by disturbance in the outflow of cerebrospinal fluid (CSF) due to excessive ICP. ${ }^{8)}$ In our patient, expansion of the ventricles was assumed to be due to obstruction of the cerebral aqueduct and disturbance of cisternal CSF flow in the posterior fossa, due to edema and swelling in the cerebellum and brainstem. Antihypertensive therapy achieved slight improvement of swelling in the brainstem and cerebellum, but the intracranial hypertension persisted, requiring extraventricular drainage and a shunt operation. A similar case of hypertensive encephalopathy with hydrocephalus has also been reported. ${ }^{9)}$

Acute hydrocephalus due to aqueductal stenosis may cause permanent visual disturbance or cerebral herniation if ICP is not controlled promptly. Improvements in hypertensive encephalopathy cannot be expected from conservative treatment of only hypertension and renal failure. Consideration must be given to extraventricular drainage or shunt operations to reduce the ICP, and control of elevated ICP due to CSF circulation disturbance is necessary.

\section{References}

1) Dinsdale $\mathrm{H}$ : Hypertensive encephalopathy, in Barnett H, Stein B, Mohr J, Yatsu F (eds): Stroke, vol 2. New York, Churchill Livingstone, 1986, pp 869-874

2) Hauser RA, Lacey DM, Knight MR: Hypertensive encephalopathy: Magnetic resonance imaging demonstration of reversible cortical and white matter lesions. Arch Neurol 45: 1078-1083, 1988

3) Katumata $Y$, Maehara T, Noda M, Shirouzu I, Shimakawa M, Hiyamuta E; Reversible CT and MR appearance. Radiat Med 11: 160-163, 1993

4) Kontos HA, Wei EP, Dietrich WD, Navari RM, Povlishock JT, Ghatak NR, Ellis EF, Patterson JL Jr: Mechanism of cerebral arteriolar abnormalities after acute hypertension. Am J Physiol 240: 511-527, 1981

5) Schwartz RB, Jones KM, Kalina P, Bajakian RL, Mantello MT, Garada B, Holman BL: Hypertensive encephalopathy: Findings on CT, MR imaging, and SPECT imaging in 14 cases. AJR Am I Roentgenol 159: $379-383,1992$

6) Strandgaard S, Paulson OB: Cerebral autoregulation Stroke 15: 413-416, 1984

7) Trommer BL, Homer D, Mikhael MA: Cerebral vasospasm and eclampsia. Stroke 19: 326-329, 1988

8) Washio M, Oh Y, Maehara F, Takeno Y, Mishima C, Fujimi S: Hydrocephalus associated with malignant 
hypertension and renal failure. Nephron 56: 222-223, 1990

9) Weingarten KL, Zimmermann RD, Pinto RS, Whelan MA: Computed tomographic changes of hypertensive encephalopathy. AJNR Am J Neuroradiol 6: 395398, 1985

10) Williams GH, Braunmald E: Hypertensive vascular disease: Malignant hypertension, in Braunwald $\mathrm{E}$, Isselbacher KJ, Petersdorf RG, Wilson JD, Martin JB,
Fauci AS (eds): Harrison's Principles of Internal Medicine, ed 11. New York, McGraw-Hill, 1987, p 1036

Address reprint requests to: H. Nakano, M.D., Department of Neurosurgery, Fujisawa City Hospital, 2-6-1 Fujisawa, Fujisawa, Kanagawa 251, Japan. 